\title{
Belphégor
}

\section{La narrazione fantastica e il mondo naturale - Introduzione}

\section{Elisa Segnini e Vittorio Frigerio}

\section{(2) OpenEdition}

\section{Journals}

\section{Edizione digitale}

URL: http://journals.openedition.org/belphegor/442

DOI: 10.4000/belphegor.442

ISSN: 1499-7185

\section{Editore}

LPCM

\section{Notizia bibliografica digitale}

Elisa Segnini et Vittorio Frigerio, «La narrazione fantastica e il mondo naturale - Introduzione », Belphégor [En ligne], 12-1 | 2014, mis en ligne le 09 juillet 2014, consulté le 19 avril 2019. URL : http:// journals.openedition.org/belphegor/442 ; DOI : 10.4000/belphegor.442

Questo documento è stato generato automaticamente il 19 aprile 2019.

\section{(c) (i) () $\Theta$}

Belphégor est mis à disposition selon les termes de la Licence Creative Commons Attribution - Pas d'Utilisation Commerciale - Pas de Modification 4.0 International. 


\title{
La narrazione fantastica e il mondo naturale - Introduzione
}

\author{
Elisa Segnini e Vittorio Frigerio
}

1 Il tema di questo numero della rivista Belphégor è stato ripreso, o quanto meno ispirato, da un simposio che portava lo stesso titolo tenutosi all'università Dalhousie nel mese di aprile 2012. Durante due giornate ricche di attività i partecipanti hanno avuto il piacere di ascoltare specialisti di varie tradizioni ed epoche discutere con entusiasmo sulla presenza del fantastico nelle opere di autori francesi, inglese, canadesi, americani, spagnoli, italiani, tedeschi, cecoslovacchi, etc. L'atmosfera conviviale, mai da sottovalutare in questo genere d'incontri, non ha potuto completamente dissipare la crescente impressione di imprecisione (non arriveremo a dire d'inquietudine, malgrado il tema dell'incontro) mano a mano che le presentazioni si susseguivano. Nonostante l'indubbio interesse delle singole analisi, è risultato sempre più chiaro che i partecipanti operavano su piani forse paralleli, ma nettamente distinti. Il concetto stesso che, idealmente, avrebbe dovuto riunirli, e che si presumeva praticamente sottinteso, era in realtà trattato in modo molto diverso e applicato a testi lontani dal punto di vista temporale, geografico e stilistico. In altre parole, non ci trovavamo davanti a una manifestazione multiforme di una nozione unica, chiamata « fantastico», ma piuttosto a varie manifestazioni, talvolta divergenti o incompatibili, di una nozione versatile che diventava sempre più difficile da circoscrivere via via che gli sforzi in tal senso si accumulavano.

2 Il seguente aneddoto può servire a presentare i parametri della questione. Durante la discussione, una tendenza costante presso i critici che introducevano le loro analisi con considerazioni generali sulla nozione di fantastico era il riferimento alla conosciutissima tesi di Tzvetan Todorov, illustrata per la prima volta nell' « Introduction à la littérature fantastique " del 1970. In vari casi, è stato tuttavia possibile constatare che il richiamo alla teoria di Todorov aveva più un valore evocativo che pratico, come se il solo nome $\mathrm{e}$ l'allusione potessero disarmare il concetto di fantastico, accantonarlo e liquidarlo prima ancora di contribuire a chiarirlo. 
3 La nozione di esitazione è stata usata frequentemente e senza esitare, ed ha permesso ai partecipanti di offrire un tributo agli dei della teoria letteraria per poi allontanarsi dagli altari con la coscienza tranquilla, continuando a rilevare nei testi studiati elementi e specificità aventi spesso ben poco, per non dire nulla, a che fare con una descrizione ortodossa del termine secondo la doxa todoroviana. Una volta stabilito che ognuno, più o meno esplicitamente, considerava il fantastico secondo il punto di vista del critico francese (ritenuto da tutte le scuole come l'approccio più semplice e fruttuoso), analisi molto diverse hanno messo in evidenza elementi «fantastici » che esulavano dal quadro presunto. In un certo senso, l'esperienza è valsa come verifica delle constatazioni avanzate da Arnaud Huftier e Bruno Bozzetto nell' opera Les Frontières du fantastique, che concludono che ciò che permetteva " a certi testi teorici francesi di passare [facilmente] le frontiere era semplicemente... la portata limitata e restrittiva di questi testi!», affermando infine: "Pare dunque che, lungi dal chiarire la situazione precedente, la traduzione e il monopolio di Todorov causino talvolta un annebbiamento opaco ${ }^{1} »$.

4 Tutto ciò non è da intendersi come critica ironica, e tantomeno come commento malevolo. L'energia dei partecipanti e le loro letture sempre animate e vivaci hanno permesso di esplorare opere che, altrimenti, avrebbero avuto ben poche possibilità di ritrovarsi l'una accanto all'altra. Hanno anche permesso di verificare l'estensione considerevole e crescente del termine fantastico nella percezione di critici che lavorano in campi molto diversi, tutti però unanimemente affascinati dalla forza d'attrazione del fantastico nella letteratura. Tutto ciò non è privo d'interesse e merita di essere oggetto di riflessione ${ }^{2}$.

5 Vorremmo suggerire che l'obbligo sentito da numerosi critici di citare, soprattutto in senso lato, la teoria di Todorov di fronte ad autori fantastici deriva da una condizione diventata talmente abituale da costituire la norma. Questo aneddoto, che non riportiamo solo per l'eventuale valore d'intrattenimento - né come avvertimento agli audaci che si propongono di organizzare altri colloqui multidisciplinari e multilingue sottostimando gli attriti possibili tra diverse culture - è in gran parte rappresentativo di una situazione reale della critica contemporanea, situazione probabilmente inevitabile quando si mettono a confronto letterature appartenenti a epoche e tradizioni che hanno poco in comune, cercando di riunirle sotto l'etichetta di un concetto che si presume operi in modo identico in ognuna di esse, malgrado le notevoli divergenze e i mezzi d'espressione artistica più disparati (letteratura di massa, letteratura alta, fumetto, cinema...).

6 Ezra Pound ha osservato che « La storia della critica letteraria è in gran parte la storia di una lotta impari per trovare una terminologia capace di definire qualcosa. Il trionfo della critica letteraria è che alcuni dei suoi termini - principalmente quelli definiti da Aristotele - mantengono ancora qualche straccio di significato ${ }^{3}$. » Aristotele, purtroppo, non si è occupato di fantastico, e benché si tenda spesso, in termini generali, a parlare del fantastico come di un "genere" a sé stante, "nessuna teoria dei generi include il fantastico ${ }^{4} »$. Lo straccio di significato al quale dovremo attaccarci dovrà dunque essere identificato altrove.

7 Il successo della teoria di Torodov è in gran parte dovuto al suo approccio astratto, che distanzia il fantastico dal contingente e ne evacua le eventuali applicazioni sociali o extraletterarie ${ }^{5}$. L'interpretazione astorica e totalizzante, che si concentra sul lettore piuttosto che su un contesto esterno all'opera e che è dunque valida per qualsiasi opera marcata dal fantastico, è stata oggetto di commenti intenzionati a restituire alla nozione di fantastico lo spessore storico trascurato. Il « sentimento del fantastico » è effettivamente presente 
in tutte le culture, ma se si desidera rendergli giustizia, è necessario "prenderne in considerazione gli effetti e situarli nel contesto dei vari periodi storici ${ }^{6}{ }$.

Secondo Roger Caillois, la cui Anthologie du fantastique ha enormemente contribuito a conferire un alone di rispettabilità al genere ${ }^{7}$, il fantastico non può che nascere $\mathrm{e}$ svilupparsi in un mondo dal quale il miracolo è stato bandito e in cui ad ogni effetto è collegata una causa rigorosamente identificabile - in altre parole, il mondo della modernità, che ha visto nascere $\mathrm{i}$ primi scrittori fantastici riconosciuti come tali (Hoffmann in Germania Nodier in Francia). Il fantastico "puro» sarebbe dunque un prodotto tipico dell'ottocento. Caillois, d'accordo su questo punto anche con Castex, considerava possibile identificare una progressione dei generi letterari che avrebbe gradualmente trasformato il meraviglioso medievale, intriso di magia, nel fantastico ottocentesco e dell'inizio del novecento, per poi, all'insegna del progresso tecnico, lasciare posto alla fantascienza. Secondo questa teoria, il fantastico si sviluppa all'interno di uno spazio umano, artificiale, urbano. Di conseguenza, tutto ciò che è familiare, rassicurante e quotidiano opera in simbiosi con il fantastico. Altri critici, avvicinandosi alla questione con maggiore licenza, hanno tentato di estendere le frontiere della dimora del fantastico. In particolare, Brian Attebery ha suggerito che « qualsiasi narrazione che include come elemento importante del suo insieme qualche trasgressione di ciò che l'autore chiaramente considera essere la legge naturale - questo è fantasy ${ }^{8}$ ". L'estensione del territorio della "legge naturale ", che arriva a coincidere con la soggettività dell'autore, de facto annulla ogni separazione arbitraria tra spazio naturale e artificiale, permettendo un ampliamento del « sentimento del fantastico » che sfuoca la frontiera tra meraviglioso, fantastico e fantascienza per evidenziare un "effetto fantastico» - nozione sulla quale insistono Huftier e Bozzetto - che può manifestarsi in diverse epoche e svariati luoghi. Charles Grivel riteneva già che se «il fantastico non si concepisce che rispetto ad una norma ", e se questa norma è la realtà, "è reale ciò che io penso che sia reale e che deve esserlo, ciò che considero tale ". L'ammissione del carattere soggettivo del contesto reale su cui si staglierebbe il fantastico portava poi il critico a precisare : "Questa realtà, beninteso, non è la natura ; ciò che esiste 'nei fatti' - come si dice - è insignificante ; al contrario, è reale ciò a cui accredito un senso. Il reale è frutto del sociale, della finalità che esso da, è ciò che si ammette come tale9.»

Sarebbe dunque impossibile l'esistenza di un «sentimento del fantastico » all'interno di un contesto naturale, esterno all'osservatore e per conseguenza perfettamente indipendente e esente da ogni sguardo soggettivo ? In un certo senso, questa verifica dell'estensione della percezione è l'oggetto di riflessione di questa raccolta.

G.K. Chesterton, in una delle discussioni paradossali in cui mescolava letteratura, sociologia e religione, evocava un fantastico dalle tonalità mistiche e suggeriva la labilità dei confini tra mondo naturale e realtà sociale. Lo faceva adottando il punto di vista di un bambino, "neutro », ingenuo, e dunque permeabile a qualsiasi influenza :

La falsa naturalezza insiste sempre sulla differenza tra il naturale e l'artificiale. La naturalezza superiore ignora questa distinzione. Agli occhi d'un bambino, l'albero e il lampione a gas sono altrettanto naturali e artificiali. 0 piuttosto, né l'uno né l'altro sono naturali, ma sono entrambi sovrannaturali. Entrambi sono splendidi e inspiegabili. Il fiore con il quale Iddio incorona l'uno, e la fiamma con la quale Sam il lampionaio incorona l'altro, sono entrambi costituiti dall'oro delle fiabe ${ }^{10}$.

11 La natura, l'extra-umano si ritrovano sullo stesso piano dell'ambiente sociale e appaiono come elementi estranei agli occhi di un osservatore innocente. L'ignoranza, nella sua 
accezione più vasta e positiva, diventa il catalizzatore che permette la nascita di un rapporto particolare con un mondo naturale colorato di fantastico. Soltanto questa condizione, paragonata nell'esempio citato al candore dell'infanzia, può permettere la manifestazione del sentimento fantastico. Quest'ultimo non è dunque un fenomeno autonomo, ma è indissolubilmente legato alla visione e alla percezione. La distanza tra il soggetto che osserva e l'oggetto osservato provoca una deformazione concettuale e attribuisce all'oggetto un carattere magico che non gli appartiene; un carattere che Chesterton considera sotto una luce poetica e che associa alla fiaba, veicolo letterario del meraviglioso e del fantastico. In circostanze diverse, lo stesso carattere magico potrebbe assumere toni meno rassicuranti. Tuttavia, perché sorga l'inquietudine, perché la paura possa cominciare a farsi lentamente sentire, occorre altro. Lo scrittore inglese rimanda l'uomo verso sé stesso, trovando che nulla è più estraneo all'osservatore del proprio riflesso :

Un uomo che percorre un viottolo la sera può osservare il palese fatto che fintanto che la natura mantiene il suo corso, non ha alcun potere su di noi. Fintanto che un albero è un albero, è un mostro dal capo enorme, con cento braccia, cento lingue e una gamba sola. Ma finché un albero è un albero, non ci spaventa per nulla. Comincia ad essere qualcosa di alieno, qualcosa di strano, solo quando ci assomiglia. Quando un albero sembra sul serio un uomo, è allora che le nostre ginocchia cominciano a tremare per davvero ${ }^{11}$.

Ed eccoci giunti al cuore della questione del rapporto tra la natura e il fantastico. La paura menzionata da Chesterton, moltiplicata dall'incredulità di fronte a un avvenimento inspiegabile, è ciò che prova Macbeth quando Birnam Wood avanza su Dunsinane, ma in questo caso il fantastico non è che un breve lampo, poiché la realtà è rapidamente ristabilita da una spiegazione razionale. Quando, nel Signore degli anelli, gli Enti di Fangorn attaccano Isengard, è la loro doppia natura, umana e vegetale, a provocare disorientamento e paura e far sì che il significato stesso della realtà venga messo in dubbio. Anche le lande percorse da Dante nella sua discesa agli inferi sono popolate da esseri ibridi, riconoscibili per le loro infrazioni alle leggi naturali, mentre il Paradiso terrestre, dotato di un carattere straordinario, è semplicemente paragonato alla pineta nei pressi Ravenna e assume così una connotazione familiare e rassicurante.

È dunque confermato che gli avvenimenti contro natura o sovrannaturali hanno bisogno, per emergere nella loro specificità, di uno sfondo ordinario contro cui stagliarsi. Questo sfondo è nella gran parte dei casi una costruzione umana, ma, ed è questa l'ipotesi di questa raccolta, può anche presentarsi sotto l'aspetto di un mondo naturale sul quale si sovrappongono - aumentando ancora il senso di stranezza - le impronte dell'umano. A provocare lo scatto del meccanismo che, grazie ad una distanza improvvisa, conferisce a un luogo tonalità insospettate e fantastiche può essere perfino la coscienza improvvisa dell'abisso che separa l'osservatore dal luogo osservato, e nel quale l'osservatore si muove, tuttavia, « naturalmente ».

Se ricorrere al sovrannaturale è una comune strategia per spiegare i fenomeni che appartengono al mondo naturale, quest'ultimo sottende alla comprensione e alla rappresentazione di ciò che si trova al di là dei suoi limiti. Nello studio consacrato al "perturbante ", Freud, nel 1919, faceva notare che la parola tedesca unheimlich è al contempo il sinonimo e il contrario di heimlich, termine che evoca conforto e familiarità, ma connota allo stesso tempo segretezza e dissimulazione. Il sovrannaturale può svilupparsi solo a partire dal naturale, ed è in effetti spesso ciò che è bello, attraente $\mathrm{e}$ sublime a trasformarsi sotto l'impulso di forze inquietanti, destando il senso d'incertezza 
nel quale si è riconosciuta la caratteristica dominante del fantastico. La natura funziona dunque in modo paradossalmente contraddittorio e complementare, rappresentando allo stesso tempo un ambito familiare e l'ignoto.

15 Così come la flora, anche la fauna può oltrepassare i limiti della descrizione tradizionale, che fa di lei una forza esterna da sottomettere, può subire una metamorfosi e ispirare identificazioni sconcertanti. La pseudo-scienza della fisiognomica (letteralmente: «la conoscenza della natura ») e i suoi addetti proponevano un'analogia tra le caratteristiche umane e le specie animali; nella letteratura fantastica, queste affinità si esprimono letteralmente quando l'umano e l'animale si trasformano l'uno nell'altro. Analogamente, le teorie di Darwin hanno ispirato alcuni racconti fantastici che, spingendo la nozione della selezione naturale fino alle sue estreme conseguenze, hanno illustrato la corrispondenza dell'ontogenesi e della filogenesi, le origini degli organismi e lo sviluppo delle specie.

16 In «La forêt du garulf dans la tradition narrative au Moyen Âge : théâtre et matrice de l'hybridation fantastique ", Loren Gonzalez osserva come la foresta medievale, nelle narrazioni del tardo dodicesimo secolo, costituisca allo stesso tempo uno spazio quotidiano e simbolico, specchio di paure ancestrali e rifugio di animali ibridi che continuano a interrogare i confini tra l'uomo e la bestia. Attraverso l'analisi di testi come Guillaume de Palerne, la Topographia Hibernica, il Lai de Melion e il Lai du Bisclavret, Gonzalez dimostra come la trasformazione del licantropo possa essere interpretata come un percorso iniziatico che porta l'eroe all'affermazione della propria individualità (e umanità) proprio attraverso l'incontro con l'alterità : prima di assumere un ruolo determinante nella vita cortese, l'eroe deve infatti affrontare ciò che rimane indomabile, asociale, selvaggio. Sebbene la visione medievale del mondo, come Gonzales sottolinea, si ponga al di là della distinzione tra naturale e soprannaturale, è possibile interpretare la foresta come spazio fantastico in quanto teatro di eventi misteriosi e inspiegabili. Spazio che rimane effimero, luogo d'incertezza ed esitazione - infatti il ritorno al genere umano, il riconoscimento dell'uomo nella bestia, non possono avere luogo se non oltre i confini del bosco. Gonzalez riprende così la nozione dell'esitazione al cuore della teoria di Todorov, ma in usandola secondo un'accezione più ampia, focalizzata soprattutto sull'« effetto fantastico » che caratterizza il momento d'incertezza tra uomo e animale, realtà e immaginario, per sfociare infine nell'accettazione del soprannaturale $\mathrm{e}$ concludersi nel meraviglioso.

17 Anche Justine Pédeflous, in « Faune diabolique et flore divine dans la légende fantastique espagnole «La azucena silvestre» (1845), de José de Zorrilla» cita la nozione dell'incertezza tra realtà e irrealtà come elemento caratteristico del fantastico, ma per andare oltre alla teoria di Todorov e rintracciare quest'esitazione nel genere della leggenda religiosa, generalmente associata al meraviglioso. Nonostante la presenza del diavolo e del divino faccia parte integrante dell'immaginario religioso, Pédeflous precisa come la loro manifestazione terrena sia comunque accompagnata dalla sorpresa, dalla paura e dal dubbio. Al cuore della leggenda di Zorrilla è infatti proprio un espediente che Todorov enumera tra $\mathrm{i}$ temi tipici del fantastico, la metafora realizzata, ovvero l'associazione dei personaggi con il mondo animale e vegetale che, nel corso della narrazione, conduce ad una vera e propria metamorfosi. Inoltre, nel momento del miracolo, il paesaggio passa da sfondo a protagonista; la natura non rispecchia più lo stato d'animo dei personaggi, ma la potenza dell'azione salvifica. Secondo Pédeflous, fantastico e il meraviglioso si distinguano solo in secondo momento, quando si tratta di 
attribuire agli eventi una spiegazione plausibile e il miracolo viene ricondotto al'intervento del divino.

La flessibilità dei confini tra fantastico e meraviglioso è messa in evidenza anche da Catherine d'Humières in « Lorsque peinture, littérature et musique suivent la piste des sirènes. L'imaginaire océanique dans De Profundis, album interactif de Miguelanxo Prado ». In quest'opera il fondale marino, che è popolato da creature ibride e da flora e fauna fantastiche (classificate, però, con precisione enciclopedica), è associato al mondo del sogno. Il mare è il luogo dell'incontro con l'Altro, ma è anche l'immagine distorta del reale e lo specchio della creazione artistica. Infatti i quadri in cui il protagonista ritrae i fondali marini costituiscono finestre, punti di intersezione tra due mondi, e a sua volta la creazione artistica non può essere ispirata che dal mondo naturale. Al centro del racconto si trova l'esperienza della metamorfosi ; come scrive l'autrice « Il faut, pour entrer vraiment dans l'histoire, que le lecteur accepte le pacte de lecture proposé par l'auteur : l'idée, récurrente dans bien des récits merveilleux ou fantastiques, que l'homme est susceptible de se transformer en une autre créature. » La differenza tra sogno e realtà, mondo naturale e creazione artistica non può essere definita, perché ognuna di queste dimensioni non esiste se non attraverso il contatto e il confronto con l'altra. In « Entre nature et surnature : les «créatures du miroir » de Joseph Sheridan Le Fanu », Isabelle Percebois esamina la raccolta In a Glass Darkly, pubblicata dallo scrittore irlandese nel 1872. L'accostamento di mondi complementari, secondo Percebois, mantiene un ruolo fondamentale nella costruzione del fantastico. Nei racconti di Le Fanu, gufi, scimmie e altri insoliti animali svolgono una funzione di mediatori, immancabilmente associati al mondo delle tenebre ma capaci di inserirsi, quasi inosservati, nella realtà del quotidiano. Percebois si sofferma sul rapporto di Le Fanu con la scrittura esoterica di Swedenborg, sull'interpretazione psicoanalitica dei racconti e su come i testi possano essere considerati un esempio di ricezione delle teorie evoluzioniste. Comune a tutte queste letture è l'idea dell'animale come immagine di alterità, materializzazione dell'innominabile, specchio delle paure più profonde. Non è tanto l'esitazione ad interessare Percebois, ma l'accostamento del fantastico al gotico e l'uso della fauna fantastica per rappresentare gli istinti bestiali che l'uomo ottocentesco cela sotto la maschera di apparente razionalità.

Restando nell'ambito del fantastico ottocentesco, ma con un radicale cambio di coordinate geografiche, Robert Summerby-Murray, in «The fantastic black panther in the writing of Charles G. D. Roberts : Forging Canadian national identity from primeval nature ", riflette sull'uso del fantastico in un racconto che descrive un animale diabolico, misterioso e incomprensibile ospitato dalle foreste canadesi. Riferendosi alla definizione di Alan Weiss, che descrive la letteratura fantastica come "Texts where fantasy and the mundane world intersect and interweave throughout a tale ", Summerby-Murray sottolinea come il racconto sia basato su un complesso rapporto tra elementi di realtà $\mathrm{e}$ finzione. L'accurata descrizione della gelida notte d'inverno è allo stesso tempo un archetipo di ambiente ostile nell'immaginario canadese; la chiesa, rintracciabile come edifico realmente esistito in New Brunswick, è anche un luogo simbolico di protezione; la foresta, dove avviene l'inspiegabile incontro con la pantera, è un luogo al di là dei confini della cristianità e della civilizzazione. Anche in questo racconto il fantastico non è che un effetto effimero che sconfina nell'allegorico e si dissolve nel momento in cui, attraversata la foresta, la pantera torna ad essere una comune fiera che viene rapidamente braccata $\mathrm{e}$ uccisa dalla comunità. 

'Cycle de Neubeourg' de Daniel Sernine", esplora la relazione tra il sublime e il fantastico nella serie di romanzi dello scrittore quebecchese. L'espediente della serie permette a Sernine di raccontare le storie di diverse generazioni che abitano la stessa regione, consentendogli di osservare con particolare precisione la trasformazione del paesaggio. Ransom sottolinea come il messaggio ecologico passi soprattutto attraverso l'evocazione di un sentimento che, seguendo la definizione di Lee Rozelle, definisce come « ecosublime» («l'émerveillement et la terreur d'une conscience surélevée du foyer écologique »), i cui effetti non sono lontani dal « piacevole terrore » descritto nel famoso trattato di Burke. Se la scoperta di tracce di civiltà sopraffatte dalla natura è nella serie associato all'Unheimlich freudiano, la percezione di fenomeni insoliti ha invece luogo al cospetto di paesaggi vasti e silenziosi ed è accompagnata da un'esperienza a metà strada tra esaltazione e terrore. L'ecosublime, come osserva Ransom, è dunque anche l'esperienza dell'allentamento dei confini che separano l'individuo dall'universo che lo circonda, la concezione di sé da ciò che viene generalmente percepito come « altro. »

Il rapporto tra fantastico e identità collettiva è ancora al cuore dell'articolo "Reinterpreting the Diaspora and the Political Violence of the Trujillo Regime: The Fantastic as a Tool for Cultural Mediation in The Brief Wondrous Life of Oscar Wao", in cui Diana Pifano esplora il complesso ritratto della diaspora dominicana che emerge dal recente romanzo di Junot Díaz (2007). Pifano presta particolare attenzione a due caratteristiche del romanzo : i continui riferimenti alla fantascienza, alla fantasy e a altre espressioni della cultura popolare nordamericana, e gli elementi soprannaturali che irrompono interrompendo la narrazione realistica, quasi storiografica. In entrambi casi, l'immaginario fantastico - che nel riflettere nozioni derivate dalla cultura nordamericana e dominicana rispecchia l'identità ibrida del protagonista - viene usato per raccontare l'indicibile, smorzando l'impatto, e rendendo quindi raccontabile, la violenza ad opera del regime di Trujillo. Pifano si sofferma in particolare sul contrasto tra le scene di violenza e il ruolo di una misteriosa mangusta dorata, immagine di una natura benigna che rende ancora più forte il contrasto con la brutalità del regime.

In « Nel bosco sacro. Realtà, finzione, magia e natura ne Il ramo d'oro di James G. Frazer » Fabiana Dimpflmeier ritorna all'esitazione di Todorov per intraprendere una lettura fantastica di un classico antropologico. Analizzando lo stile e le modalità di narrazione di Frazer, Dimpflmeier dimostra come l'antropologo «racconti» da vero a proprio romanziere, servendosi di un « orrendo rituale » come incipit e dei boschi sacri e del culto degli alberi come leitmotiv. L'autrice evidenzia come lo scopo di Frazer non sia tanto usare la scienza per spiegare le credenze che fanno parte integrante dell'immaginario dell'uomo primitivo, ma portare il lettore a confrontarsi con la sussistenza della magia e dell'irrazionale nella società contemporanea. Per riuscire nel suo intento, Frazer continua a evocare l'indecisione tra sogno e veglia, realtà e illusione. « Siamo nella categoria dello strano fantastico", scrive Dimpflmeier, "dove avvenimenti insoliti ricevono una spiegazione razionale, o dello 'strano puro' al confine fra fantastico e letteratura ". L'antropologo riesce così a descrivere « la scoperta del meraviglioso, magico, mistico e sottile incanto emanato dalla vegetazione assieme allo svelamento del suo lato più inquietante e selvaggio.»

Infine François-Ronan Dubois, in «La forêt et ses habitants fantastiques dans les séries télévisées anglophones ", esplora il ruolo delle foreste in numerose serie televisive tra cui The X-Files, Buffy the Vampire Slayer and Supernatural. Dubois identifica tre principali 
categorie d'analisi : il caso della foresta come rifugio di creature mostruose, spesso frutto di una metamorfosi che riduce l'umano all'animale; il caso in la foresta, creando una barriera tra la civilizzazione e il mondo selvaggio, fa emergere negli esseri umani tendenze inumane che li spingono ad infrangere tabù come l'omicidio e l'incesto ; il caso in cui la foresta stessa può essere considerata un'entità vivente, incarnazione polimorfa del mondo naturale. Soffermandosi su episodi che mettono in scena licantropi, chupacabra , wendigo e alberi animati, Dubois osserva come la foresta continui ad essere caratterizzata come un luogo alienato e alienante, spazio che, nel contenere tutto ciò che è ibrido e istintivo, interroga anche i limiti della razionalità umana e costringe l'umanità a confrontarsi con una componente atavica. Proprio nello smorzarsi dei confini tra l'umanità e animalità Dubois ritrova una traccia dell'esitazione di Todorov : « Le caractère surnaturel des créature évoqués, par fois mal déterminé (c'est le cas propre de l'indécision fantastique), naît d'une rupture dans l'ordre hermétique des choses, c'est à dire dans l'organisation logique du monde qui maintient de part et d'autre d'une frontière fermement définie l'humain et l'animal ».

Con questa riflessione, Dubois si riallaccia al primo articolo di questa raccolta, dimostrando come la rappresentazione del mondo naturale, nelle fiction fantastica, sia in fondo poco cambiato dall'epoca medievale e come, lungi dall'avere raggiunto un equilibro, il nostro rapporto il mondo naturale rimanga fondamentalmente ambiguo e inquietante.

\section{NOTE}

1. «[...] le franchissement des frontières de certains textes théoriques français, c'est tout simplement... le cadre limité et limitatif de ces textes !» [...] « Il apparaît donc que, loin d'éclaircir la situation préalable, la traduction et la mainmise de Todorov apportent des brouillages parfois opaques ». Bozzetto, Roger e Arnaud Huftier. Les Frontières du fantastique. Approches de l'impensable en littérature. Valenciennes, Presses Universitaires de Valenciennes, 2004, p. 16 e 20.

2. Che il lettore si rassicuri : la collezione che gli è qui proposta è frutto di una raccolta ulteriore di testi, tra i quali alcuni presentati al colloquio e vari altri ottenuti attraverso una chiamata di contributi.

3. «The history of literary criticism is largely the history of a vain struggle to find a terminology which will define something. The triumph of literary criticism is that certain of its terms chiefly those defined by Aristotle - still retain some shreds of meaning. » Pound, Ezra. The Spirit of Romance. New York, New Directions, 1968, p.13.

4. «[...] aucune théorie des genres n'inclut le fantastique ». Steinmetz, Jean-Luc. La littérature fantastique. Paris, Que Sais-je ?, 1990, p.4.

5. Si veda a riguardo, tra l'altro, l'articolo di Giulio Lepschy, « Aspetti linguistici del fantastico ». In Gli Universi del fantastico. A cura di Vittore Branca e Carlo Ossola. Firenze, Vallecchi Editore, 1988, pp. 147-186.

6. « [...] il s'agit d'envisager les effets et de les situer dans le cadre de périodes historiques». Bozzetto, Roger. Fantastique et mythologies modernes. Aix-en-Provence: Publications de l'Université d'Aix-en-Provence, 2007, p. 226. 
7. Caillois, Roger. Anthologie du fantastique. Paris : Gallimard, 1966, 2 vol.

8. «Any narrative which includes as a significant part of its make-up some violation of what the author clearly believes to be natural law - that is fantasy ». Attebery, Brian. The Fantasy Tradition in American Literature. Bloomington : Indiana University Press, 1980, p. 2. Corsivo nostro.

9. «Le fantastique ne se conçoit que par rapport à une norme. » [...] « est réel ce que je pense qu'il l'est et qu'il le doit, ce que je retiens comme tel.» [...] «Ce réel, bien sûr, n'est pas nature; ce qui existe 'en fait' - comme on dit - est insignifiant; au contraire, est réel ce que j'accrédite d'un sens. Le réel est issu du social, de la finalité qui lui est propre, il est ce qui s'admet. » Grivel, Charles. Le Fantastique. Mannheim : Mannheim-Analytiques, 1983, p. 7-8. Corsivo dell'autore.

10. The false type of naturalness harps always on the distinction between the natural and the artificial. The higher kind of naturalness ignores that distinction. To the child the tree and the lamp-post are as natural and as artificial as each other; or rather, neither of them are natural but both supernatural. For both are splendid and unexplained. The flower with which God crowns the one, and the flame with which Sam the lamplighter crowns the other, are equally of the gold of fairy-tales.

Chesterton, G.K. Heretics. New York : John Lane Company, 1905, p.139.

11. «For a man walking down a late at night can see the conspicuous fact that as long as nature keeps to her own course, she has no power with us at all. As long as a tree is a tree, it is a topheavy monster with a hundred arms, a thousand tongues, and only one leg. But so long as a tree is a tree, it does not frighten us at all. It begins to be something alien, to be something strange, only when it looks like ourselves. When a tree really looks like a man our knees knock under us ". Chesterton, p. 152. 